\title{
INTERNATIONAL JOURNAL OF SCIENTIFIC RESEARCH
}

\section{COMPARATIVE ANALYSIS BETWEEN ULTRASONIC TIPS AND HAND INSTRUMENTS ON THE CLINICAL PARAMETERS OF PERIODONTAL DISEASE.}

\begin{tabular}{ll}
\hline Dental Science & \\
$\begin{array}{l}\text { Dr Avadhesh } \\
\text { Kumar }\end{array}$ & $\begin{array}{l}\text { M.D.S., Professor, Department of Periodontology, Career P.G. Institute of Dental } \\
\text { Sciences \& Hospital, I. I.M. Road, Lucknow (U.P.), India. }\end{array}$ \\
\hline Dr Jaya Dixit & $\begin{array}{l}\text { M.D.S., Ex-Professor and Head, Department of Periodontology, Faculty of Dental } \\
\text { Sciences, King George's Medical University, Lucknow, (U.P.), India. }\end{array}$ \\
\hline Dr Nand Lal & $\begin{array}{l}\text { M.D.S., Professor and Head, Department of Periodontology, Faculty of Dental Sciences, } \\
\text { King George's Medical University, Lucknow, (U.P.), India. }\end{array}$ \\
\hline $\begin{array}{l}\text { Dr Shalini } \\
\text { Kaushal* }\end{array}$ & $\begin{array}{l}\text { M.D.S., Professor, Department of Periodontology, Faculty of Dental Sciences, King } \\
\text { George's Medical University, Lucknow, (U.P.), India. *Corresponding Author }\end{array}$ \\
\hline
\end{tabular}

\section{ABSTRACT}

Aims: To compare hand versus ultrasonic scaling and root planning (SRP) on the clinical parameters of periodontal disease and to assess left over calculus by open flap approach.

Methods And Material: The study sample consisted of random selection of 30 patients with the evidence of chronic inflammatory periodontal disease. Two groups were made in each patient by split mouth design. Pre treatment measurements recorded were plaque index (PI), calculus index(CI) and probing pocket depth(PPD). Post treatment measurements recorded were PI, PPD and left over calculus after flap elevation Results: In hand instrumentation group (HIG),, mean percentage of plaque score was slightly lesser as compared to ultrasonic but not significant. Overall mean reduction in PPD after instrumentation in HIG was greater as compared to ultrasonic, but it was not statistically significant ( $\mathrm{p}>0.05$ ). The percentage efficiency in removal of calculus of HIG was greater compared to ultrasonic but it was also not statistically significant ( $p>0.05$ ). There was no significant difference between HIG and ultrasonic for number of sites with residual calculus and moreover no. of sites increased apically.

Conclusions: There was significant post-treatment change in the clinical parameters taken in the present clinical study in both HIG \& UIG and also slightly more favourable with HIG but not statistically significant.

\section{KEYWORDS}

Open flap approach, periodontal disease, scaling and root planing ,ultrasonic

\section{INTRODUCTION}

Periodontal therapy consists of treatment modalities aimed at arresting infection and maintaining a healthy periodontium. The periodic mechanical removal of subgingival microbial biofilms (bacterial plaque) is essential for controlling inflammatory periodontal diseases, because disease causing bacteria can repopulate pockets within weeks following active therapy Sbordone ${ }^{[1]}$

Scaling and root planing whether by hand instruments or ultrasonic instruments have consistently been shown to be one of the most effective means of treating periodontal diseases. Several studies have been performed comparing the two with varying results. In several studies utilizing the dissection microscope Schaffer ${ }^{[2]}$, Belting and Spjut ${ }^{[3]}$ and the profilometer Pameijer et al. ${ }^{[4]}$, curettes (hand instrumentation) produced roots surfaces which were smoother than those produced by ultrasonic instrumentation. Although both instruments appeared to be effective in removing calculus, but the question is which method is more effective in complete removal of subgingival calculus when visualized clinically.

\section{MATERIALSAND METHODS}

The present study was conducted in the Department of Periodont0logy,KGMU, Lucknow. Thirty patients between the age group of 20 and 50 years were selected from the Outpatient Department clinic, after obtaining approval from ethical committee, irrespective of the sex and socio-economic status. Two groups (HIG hand instrumentation group and UIG - ultrasonic instrumentation group) were made in each patient by split mouth design. Pre-treatment measurements recorded were plaque index, calculus index and probing pocket depth. Post treatment measurements recorded were plaque index, probing pocket depth and left over calculus after flap elevation.

The criterion for inclusion was systemically healthy subjects with the evidence of chronic inflammatory periodontal disease and probing pocket depth $>4 \mathrm{~mm}$ after scaling and root planning and subjects with calculus score $>2$ and no missing maxillary anterior teeth (Fig.1).Subjects with periodontal surgery or medications for last six months, surface caries or any subgingival restoration, smokers and pregnant women were not included in the study.

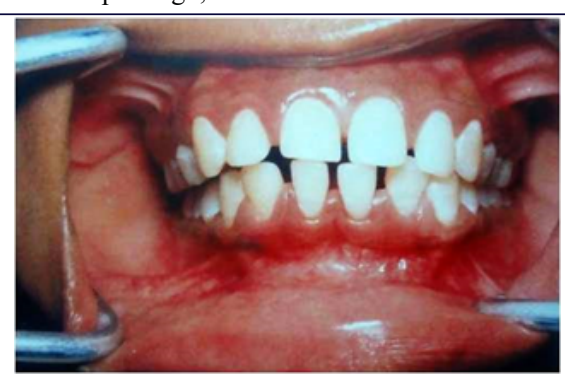

Fig.1 Preoperative.

Thorough scaling and root planing was done. On one side it was performed with the help of Satelac P5 ultrasonic scaler and on the other side by using hand instruments (Gracey curettes), until the root surface felt smooth and glass hard to the explorer. The subjects were instructed for good oral hygiene maintenance.

Two weeks after thorough scaling and root planing, subjects were recalled for surgical procedure. All the instruments used in the surgical procedure were sterilized. Surgical procedure in all the subjects was performed under local anesthesia with a solution of $2 \%$ Lignocaine with 1:1,00,000 adrenaline. Before nerve block or local infiltration, each subject was tested for sensitivity. After adequate anesthesia in the surgical area, a full thickness periosteal flap was elevated to gain access to the root surfaces (Fig.2). After removal of granulation tissue, the root surfaces were gently irrigated with sterile saline solution. Using sterile surgical gauze moistened with sterile saline, each root surface was visually examined for the presence or absence of residual calculus (RC). Aids to assist in the detection of calculus beyond the visual level were not attempted in this clinical study. When calculus was detected, its location on the root and distance in millimeters from cementoenamel junction (CEJ) were determined and recorded. The $\mathrm{RC}$ was removed and root surfaces were planed and surgical area was thoroughly debrided. The flaps were repositioned and sutured by using 3-0 silk suture material and periodontal dressing was given.

After surgery, medications (Doxycycline $100 \mathrm{mg}$ twice on the first day 
followed by $100 \mathrm{mg}$ once daily for 5 days. Nimesulide $100 \mathrm{mg}$ twice daily and B-Complex, 1 capsule once daily for 5 days) and postoperative instructions were given. Chlorhexidine mouthwash was prescribed twice daily for three weeks.. The subjects were recalled after 7 days for the removal of periodontal dressings and sutures (Fig.3).

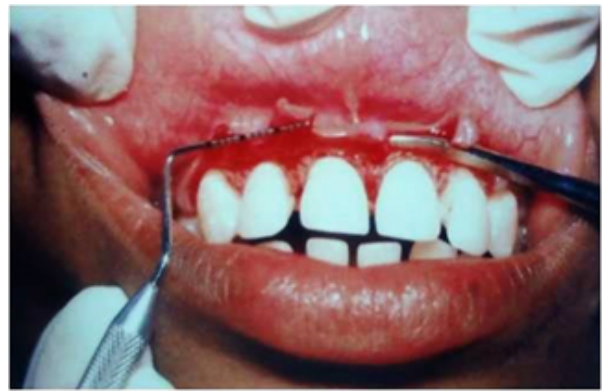

Fig. 2 - Periodontal Flap Reflected To See Residual Calculus.

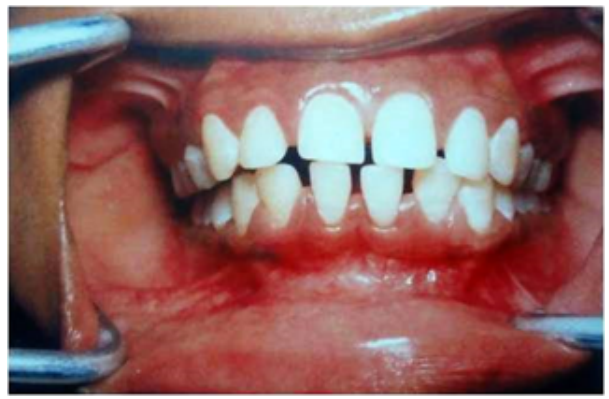

Fig.3 Postoperative.

\section{Clinical Parameters}

Plaque Index by Silness and Löe.

' 0 ' = No plaque in the gingival area

' 1 ' = A thin film of plaque adhering to the free gingival margin and adjacent area of the tooth. The plaque may be recognized only by running a probe across the tooth surface.

'2' = Moderate accumulation of soft deposits within the gingival pocket and on the gingival margin and/or adjacent tooth surface that can be seen by the naked eye.

' 3 ' = Abundance of soft matter within the gingival pocket and/or on the gingival margin and adjacent tooth surface.

\section{Calculus Index}

' 0 ' = Absence of calculus

' 1 ' = Supragingival calculus extending only slightly below the free gingival margin (not more than $1 \mathrm{~mm}$ )

'2' = Moderate amount of supragingival and subgingival calculus or subgingival calculus alone.

'3'=An abundance of supragingival and subgingival calculus.

\section{Probing Pocket Depth}

Probing Pocket depth was recorded in millimeters with the help of UNC 15 periodontal probe from gingival margin to the base of the pocket at six locations

\section{Left Over Calculus}

After recording PI and PPD, a full thickness flap was

elevated to gain access to the root surface and RC was scored according to following criteria.

' 0 ' = No calculus

' 1 '= Calculus upto $2 \mathrm{~mm}$ from CEJ

' 2 ' = Calculus upto $4 \mathrm{~mm}$ from CEJ

' 3 ' $=$ Calculus $>4 \mathrm{~mm}$ from $\mathrm{CEJ}$

\section{Statistical Analysis}

The statistical analysis of the data was done using Statistical Package for Social Sciences Version 10.0 (SPSS 10.0). The mean of each experimental group was done to depict the central tendency of that group. Standard Deviation: Standard deviation denoted the degree of variation from the central tendency. Paired ' $t$ ' test was done to see the difference between pre and post-experiment values. As the design of the present study was experimental, the differences between groups were calculated using Student's 't' test for independent samples. Chisquare test was used to see the association between two groups.

The significance levels were graded as follows : $\mathrm{p}<0.05$ - Significant $(95 \%$ level of significance $)$ $\mathrm{p}<0.01$ - Highly significant ( $99 \%$ level of significance) $\mathrm{p}<0.001$ - Very highly significant $(99.9 \%$ level of significance $)$

\section{RESULTS}

The study was conducted on the 1080 sites for PPD and calculus score and 720 sites for PI in 30 subjects. Out of these sites, $50 \%$ sites were instrumented with hand instruments and remaining $50 \%$ sites with ultrasonic instruments. The efficiency of both the instruments was compared with respect to PI, CI and PPD. In both the groups, there was reduction in mean percentage of PI which was statistically significant. In HIG, there was reduction from $80.83 \%$ to $17.50 \%$ and in UIG, from $78.61 \%$ to $20.00 \%$ (Graph 1). There was overall mean reduction in PPD of $1.5 \mathrm{~mm}$ after hand instrumentation and after ultrasonic instrumentation it was $1.36 \mathrm{~mm}$. (Graph 2).

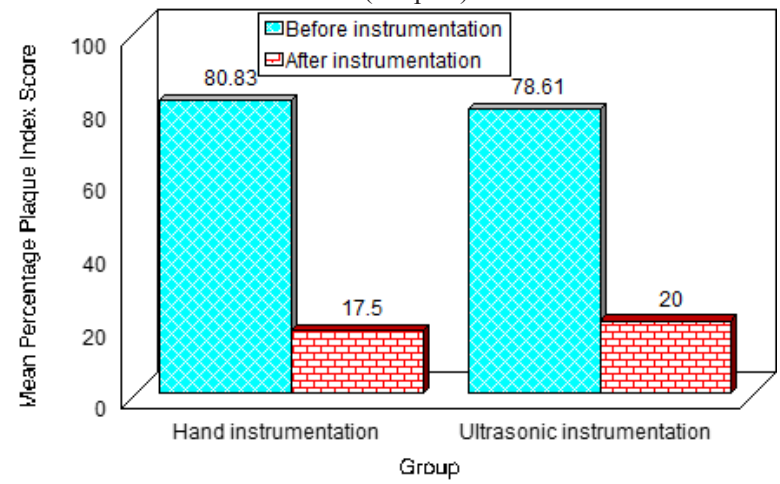

Graph 1 Comparison Of Mean Percentage Of Plaque Score Before And After Instrumentation.

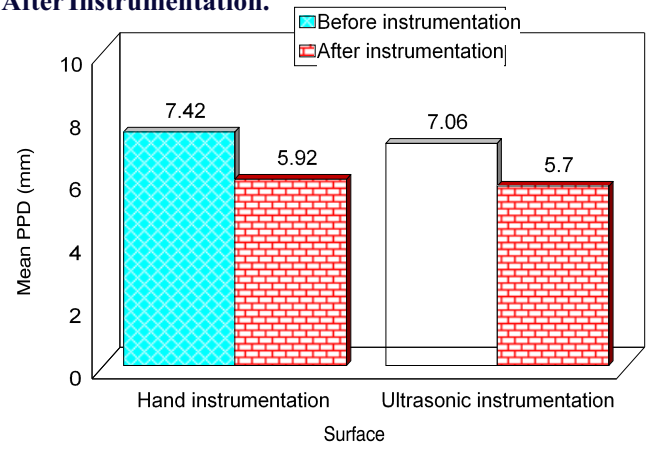

\section{Graph 2 Mean Reduction In PPD After Instrumentation.}

In HIG, 42 sites with RC were noticed and in ultrasonic instrumentation, 51 sites. (Graph 3). Table 1 shows comparison of percentage efficiency of hand instruments \& ultrasonic tip in the removal of calculus. An increase in the percentage of $\mathrm{RC}$ was noticed with increase in ppd and also presence of $\mathrm{RC}$ was more as distance increased from CEJ apically (Table 2). There was significant posttreatment change in the clinical parameters taken in the present clinical study in both HIG \& UIG and also slightly more favourable with HIG but not statistically significant.

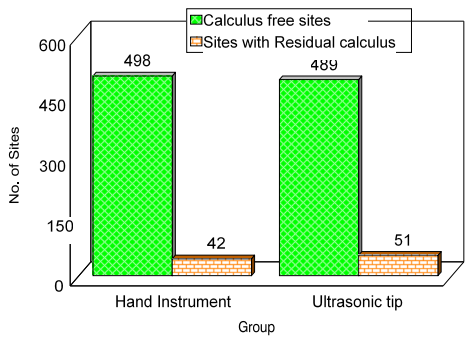

Graph 3 No. Of Sites With RC In Hand And Ultrasonically Instrumented Groups. 
Table1: Comparison Of Percentage Efficiency Of Hand Instruments \& Ultrasonic Tip In The Removal Of Calculus.

\begin{tabular}{|l|l|l|l|}
\hline Instruments & $\begin{array}{l}\text { Total No. of } \\
\text { sites }\end{array}$ & $\begin{array}{l}\text { No. of Calculus } \\
\text { free sites }\end{array}$ & Percentage Efficiency \\
\hline Hand instruments & 540 & 498 & 92.33 \\
\hline Ultrasonic tip & 540 & 489 & 90.56 \\
\hline $\begin{array}{l}\text { Comparison of } \\
\text { percentage efficiency } \\
\text { (Hand instrumentation } \\
\text { vs Ultrasonic tip) }\end{array}$ & $\chi^{2}=0.31, p=0.57$ & \\
\hline
\end{tabular}

Table 2 : No. of sites with Residual Calculus in relation to distance (in $\mathrm{mm}$ ) from CEJ to apical border of residual calculus.

\begin{tabular}{|l|l|l|}
\hline \multirow{2}{*}{ Distance (in mm) } & \multicolumn{2}{|l|}{ No. of sites with residual calculus } \\
\cline { 2 - 3 } & HIG & UIG \\
\hline Upto $1 \mathrm{~mm}$ & 9 & 12 \\
\hline$>1$ to $2 \mathrm{~mm}$ & 6 & 6 \\
\hline$>2$ to $3 \mathrm{~mm}$ & 6 & 6 \\
\hline$>3$ to $4 \mathrm{~mm}$ & 12 & 12 \\
\hline$>4 \mathrm{~mm}$ & 12 & 15 \\
\hline
\end{tabular}

\section{DISCUSSION}

The goal of periodontal therapy is to restore health and function of the periodontium and maintain the natural dentition for lifetime. Correlation of supragingival plaque and calculus with gingivitis and periodontitis has been demonstrated in several studies, Lindhe et al. ${ }^{[3]}$ and Loe et al. ${ }^{[6]}$

Scaling and root planing are widely used techniques in periodontal therapy to remove irritants from the surfaces of the teeth and also to reduce tooth surface roughness which may facilitate the accumulation of irritants, Waerhaug ${ }^{[7]}$. The use of ultrasonic or hand instruments for therapy has been critically evaluated, Garnick ${ }^{[8]}$ and Zitterbart ${ }^{[9]}$. Both therapies have been shown to be effective in reducing supra- and subgingival plaque, Breininger et al. ${ }^{[10]}$ and removing endotoxins from root surfaces, Checchi et al. ${ }^{[11]}$. One advantage of the ultrasonic scaler is the associated flow of water, which besides acting as a medium for tip cooling and for cavitational activity, also serve to clear the site of debris and to improve the field of vision during non-surgical, Badersten et al ${ }^{[12]}$ or surgical root surface debridement. A great number of studies have shown that despite the best efforts of different clinicians to thoroughly plane the root surface, considerable amount of calculus remained, although the surface was clinically smooth specially in surface defects Waerhaug ${ }^{[13]}$.

In the past, periodontal debridement (scaling and root planing) was primarily performed with hand instruments since sonic and ultrasonic scalers originally were designed for gross scaling and removal of supragingival calculus and stains Johnson \& Wilson ${ }^{[14]}$. More recently, these power-driven instruments have been modified to have smaller diameter tips and longer working lengths, thereby providing better access to deep probing sites and more efficient subgingival instrumentation Holbrook \& Low $^{[15]}$. In past, several studies have shown contradictory results regarding the efficacy of hand versus ultrasonic instrumentation. The present study was therefore conducted to determine which instrumentation is more effective \& results in less amount of residual calculus, less score of PI \& greater reduction in PPD

In the present study, maxilliary anterior teeth were selected for instrumentation by split mouth technique. Subjects with PPD $>4 \mathrm{~mm}$ were selected because various studies have shown that complete removal of calculus is almost $100 \%$ possible in PPD $<3 \mathrm{~mm}$. Stambaugh et al. ${ }^{16]}$ reported that pocket areas more apical than $3.7 \mathrm{~mm}$ could not be effectively instrumented. Subjects with subgingival calculus were included in the study to observe presence or absence of residual calculus after flap elevation.

For PI \& PPD, the reduced scores were recorded after 2 weeks of instrumentation because Kon et al. ${ }^{[17]}$ reported that in the 12 day specimen the sulcus had returned to a normal and healthy condition. PI was recorded according to criteria of Silness \& Löe ${ }^{[18]}$ for plaque score. Probing pocket depths were recorded by UNC 15 periodontal probe. Evaluation of RC was done by visual inspection after elevation of flap. The distance in $\mathrm{mm}$. from CEJ to most apical border of residual calculus was measured $\&$ different scores were given. This method of scoring RC was different from other authors Chan YK et al. ${ }^{[19]}$ but was similar to the study by Gellin et al. ${ }^{[20]}$

In the present clinical study, mean percentage of plaque score was reduced from 80.83 to 17.50 in HIG \& in UIG, from 78.61 to 20.00 which was in agreement with the study done by Laurell et al. ${ }^{[21]}$ who reported $75 \%$ reduction in sites with plaque by hand instrumentation \& $77 \%$ by ultrasonic scalers. Boretti et al ${ }^{[22]}$ reported $28 \%$ reduction in sites with plaque by hand instrumentation \& $19 \%$, with ultrasonic scalers. However, when both the groups were analyzed to determine whether a significant difference existed between HIG \& UIG in removal of plaque, analysis showed no significant difference between the two methods which was in agreement with results shown by Copulos et al..$^{[23]}$ and Boretti et al. ${ }^{[22]}$.

For calculus score, out of 540 sites, 42 sites with residual calculus were noticed in HIG, \& in UIG, 51 sites. Therefore percentage efficiency of hand instruments was $92.33 \%$ \& of ultrasonic scaler was $90.56 \%$, which appears in agreement with results of Barnes \& Schaffer ${ }^{[2]}$ who reported $94.6 \%$ of calculus was removed by curettes. Stende \& Schaffer ${ }^{[25]}$ also reported $88.16 \%$ efficiency of ultrasonic scalers and $90.48 \%$ of hand instruments. Jones WA \& O' Leary ${ }^{[26]}$ reported $18.75 \%$ of the visible flecks of calculus on root planed surface detected after post extraction examination. Gellin et al. ${ }^{[20]}$ reported $26.8 \%$ of the residual calculus by measuring extent of most apical border of residual calculus in $\mathrm{mm}$ from CEJ.

An increase in the percentage of $\mathrm{RC}$ was noticed with increase in probing pocket depth and also presence of RC was more as distance increased from CEJ apically. In HIG, percentage of sites with RC in PPD $<4 \mathrm{~mm}$ was $14.28 \%$ and in UIG it was $17.64 \%$. In PPD $>4 \mathrm{~mm}$, in HIG it was $85.7 \%$ and in UIG it was $82.35 \%$. This was in corroboration with studies of Rabbani et al. ${ }^{[27]}$ who found high correlation between percentage of RC \& PPD.

For PPD, in HIG, the mean reduction range observed was from 1.2 to $2.0 \mathrm{~mm}$. In UIG, the range varied from 1.0 to $2.0 \mathrm{~mm}$. The overall mean reduction in HIG was $1.50 \mathrm{~mm}$ and in UIG it was $1.36 \mathrm{~mm}$. Boretti et al ${ }^{[22]}$ reported significant decrease in probing depth by manual and ultrasonic instrumentation with no significant difference when results of both the instrumentation groups were compared. The similar results were obtained in the present clinical study also. The previous studies by Bandersten et al. ${ }^{[28]}$ and Laurell et al ${ }^{[21]}$ compared the powered and hand instrumentation for periodontal scaling and root planing and reported similar reduction in probing depth, similar results were obtained in the present study. Overall mean reduction in PPD was 1.5 $\mathrm{mm}$ in HIG and $1.36 \mathrm{~mm}$ in UIG which was in agreement with 27 studies compiled by 1996 World Workshop in Periodontics which indicated $1.29 \mathrm{~mm}$ reduction in moderate pockets and $2.16 \mathrm{~mm}$ in deep pockets. Copulos et al.$^{[23]}$ also reported similar results.

\section{CONCLUSION}

There was significant change in the clinical parameters taken in the present clinical study in both HIG \& UIG and also slightly more favorable with HIG but not statistically significant. As the distance from CEJ increased apically, number of sites with RC also increased in both the groups but no significant difference between HIG and UIG for number of sites with RC. These findings led to several interesting questions or speculations. Considering the inadequacies of present methods of instrumentation, could one expect to find complete calculus removal? If, then, $\mathrm{RC}$ is to be expected, obvious questions arise. How much calculus can be left and the tissue remain healthy? Further study must be therefore undertaken in this direction.

\section{REFERENCES}

1. Sbordone, L., Ramaglia, L., Guletta, E., \& Iacono, V. (1990). Recolonization of the subgingival microflora after scaling and root planing in human periodontitis. Journal of Periodontology, 61(9), 579-584.

2. Schaffer, E.M. (1956). Histological results of root curettage of human teeth. Journal of Periodontology, 27(4), 296.

Belting, C.M. \& Spjut. (1964). Effects of high speed periodontal instruments on the roo surface during subgingival calculus removal. Journal of American Dental Association, $69,578-584$

4. Pameijer, C.H., Stallard, R.E. \& Hiep, N. (1972). Surface characteristics of teeth following periodontal instrumentation: a scanning electron microscope study. Journal of Periodontology, 43(10), 628-633.

5. Lindhe, J., Hamp, S. \& Loe, H. (1973). Experimental periodontitis in the Beagle dog. Journal of Periodontal Research, 8(1), 1-10.

6. Loe, H., Theilade, E. \& Jensen, S.B. (1965). Experimental gingivitis in man. Journal of Periodontology, 36,177-187.

Waerhaug, J. (1956) Effect of rough surfaces upon gingival tissues. Journal of Dental Research, 35, 323-325.

Garnick, J.J., (1989). A SEM study of root surfaces and subgingival bacteria after hand and ultrasonic instrumentation. Journal of Periodontology, 60, 441-447. 
9. Zitterbart, P.A. (1987). Effectiveness of ultrasonic scalers. A literature review. General Dentistry, 35,295-297.

10. Breininger, D.R. O'Leary, T.J. \& Blumenshine, R.V.H.(1987). Comparative effectiveness of ultrasonic and hand scaling for the removal subgingival plaque and calculus. Journal of Periodontology, 58(11), 9-11.

11. Checchi, L. \& Pelliccioni, G.A.(1988). Hand versus ultrasonic instrumentation in the removal of endotoxins from root surfaces in vitro. Journal of Periodontology, 59, 398-402.

12. Badersten, A., Nilveus, R. \& Egelberg, J. (1985). Effect of non-surgical periodontal therapy (IV) .Operator visibility. Journal of Clinical Periodontology, 12, 190-200.

13. Waerhaug, J. (1978b). Healing of the dentoepithelial junction following subgingival plaque. II. As observed on extracted teeth. Journal of Periodontology, 49, 119-134.

14. Johnson, W.N. \& Wilson, J.R. (1957) Application of ultrasonic dental unit to scaling procedures. Journal of Periodontology, 28, 264-271.

15. Holbrook, T.E. \& Low, S.B (1994). Power-driven scaling and polishing instruments. In: Clark JW ed. Clark's Clinical Dentistry, Philadelphia: JB Lippincott, 3,1-24.

16. Stambaugh, R.V., Dragoo, M., Smith, D.M., \& Carasali, L. (1981). The limits of the subgingival scaling. International Journal of Periodontics Restorative Dentistry, 1(5):30-41

7. Kon, S., Novaes, A.B., Ruben, M.P. \& Goldman, H.M. (1969). Visualization of microvascularization of the healing periodontal wound. II. Curettage. Journal of Periodontology, 40(2),96-105

18. Silness, P.\& Löe, H.(1964). Periodontal disease in pregnancy. Acta Odontologica Scandinavica, 22(1), 121-135.

19. Chan, Y.K., Needleman, I.G. \& Clifford, L.R. (2000) Comparison of four methods of assessing root surface debridement. Journal of Periodontology, 71, 385-393.

20. Gellin, R.G. Miller, M.C. Javed, T. Engler, W. \& Mishkin, D.J. (1986). The effectiveness of the Titan-S sonic scaler versus curettes in the removal of subgingival calculus: A human surgical evaluation. Journal of Periodontology, 57(11), 672-680.

21. Laurell, L. \& Pettersson, B. (1988). Periodontal healing after treatment with either the Titan-S sonic scaler or hand instruments. Swedish Dental Journal, 12, 187-192.

22. Boretti, G., Zappa, U., Graf, H. \& Case, D.(1995). Short-term effects of phase I therapy on crevicular cell populations. Journal of Periodontology, 66, 235-240

23. Copulos, T.A., Low, S.B., Walker, C.B., Trebilcock, Y.Y. \& Hefti AF.(1993).Comparative analysis between a modified tip and hand instruments on clinical parameters of periodontal disease. Journal of Periodontology, 64, 694-700.

24. Barnes, J.E. \& Schaffer, E.M. (1960). Subgingival root planing: A comparison using files, hoes and curettes. Journal of Periodontology, 35, 300-303.

25. Stende, G.W. \& Schaffer, E.M. (1961). Comparison of ultrasonic and hand scaling. Journal of Periodontology, 32,312-314.

26. Jones, W.A. \& O'Leary, T.J. (1978). The effectiveness of in vivo root planing in removing bacterial endotoxin from the roots of periodontically involved teeth. Journal of Periodontology, 49337-342.

27. Rabbani, G.M., Ash, M.M. \& Caffesse, R.G. (1981).The effectiveness of subgingival scaling and root planing in calculus removal. Journal of Periodontology, 52, 119-123.

28. Badersten, A., Nilveus, R. \& Egelbery, J. (1984). Effects of non-surgical periodontal therapy II. Severely advanced periodontitis. Journal of Clinical Periodontology, 11: 63-76. 